\title{
INTERPRETING FITTING PARAMETERS OF TEMPERATURE DEPENDENCE OF DARK CURRENTS IN SOME CCDS
}

\author{
E. Bodegom, R. Widenhorn, D. A. Iordache* \\ Department of Physics, Portland State University, Portland, Oregon, USA, OR97207 \\ E-mail: Bodegom@pdx.edu \\ *Physics Department, University "Politehnica", Splaiul Independenței, Bucharest, Romania \\ E-mail: daniordache2003@yahoo.com
}

\begin{abstract}
The experimental results concerning the temperature dependence of the dark currents in some charge-coupled devices (CCDs) were analyzed. It was found that the used theoretical model allows: (i) the evaluation of the lowest limit of experimental errors (involving the systematic ones), (ii) the study of Meyer-Neldel relations, pointing out the high correlation of diffusion dark currents with the energy gap $E_{g}$, unlike the corresponding weak correlation of depletion dark currents. (iii) rather accurate assignments of the obtained values of deep-level traps energies to some specific impurities.

Keywords: Charge-Coupled Devices, Dark Currents. Diffusion Currents, Depletion Currents, Meyer-Neldel Relations, Deep-Level Traps.
\end{abstract}

\section{INTRODUCTION}

It is well-known [1]-[3] the necessity of the previous estimation (before the fitting parameters evaluation) both of errors corresponding to the individual values dispersion (an easy task. leading to a zero-order approximation of experimental errors) and of the "systematic" errors (due to the specific features of the used experimental installation).

In order to obtain some evaluations of the true errors (involving the systematic ones, whose accurate determination is rather difficult, sometimes practically impossible), we studied the compatibility of the predictions of different theoretical models of dark currents relative to experimental data. The lowest limit of the true experimental errors was evaluated so, being compared permanently with the description errors of different theoretical models of dark currents. The obtained values of fitting parameters were analyzed, in order to find their physical meaning.

\section{EXPERIMENTAL PART}

The experimental procedures used for the determination of dark currents in the charge-coupled devices (CCD) were described by work [4]. The dark currents obey (as many thermally activated processes) the Arrhenius law: $D e^{-}=D e_{0}^{-} \exp (-\Delta E / k T)$,

their values increasing exponentially towards higher temperatures. According to the experimental results reported by work [5], the average values of the dark currents for 20 randomly chosen pixels were only 0.00978 counts $/ \mathrm{s}$ at $T=222 . K, 0.0387$ counts $/ \mathrm{s}$ at $T=$ $232 \mathrm{~K}$, reaching the level of 223.433 counts $/ \mathrm{s}$ at $T=291$ $K$. That is why the relative standard (square mean) errors of the individual values were rather large àt low temperatures: $35.85 \%$ at $T=222 K$ and $9.45 \%$ at $T=232 K$, decreasing quickly: $3.53 \%(242 K), 1.03 \%$ $(252 K), 0.486 \%(262 K), 0.233 \%(271 K$ and $281 K$ respectively), $0.297 \%(291 K)$. Eliminating the lowest studied temperatures (222 and $232 K$. in order to ensure a good accuracy of fitting parameters evaluations). we have found an average relative standard error (due to the dispersion of experimental individual values) of $1.525 \%$ for the 6 highest temperatures $(242 \ldots 291 K)$. This value represents the zero-order approximation of the experimental errors.

Some unwanted implications of the dark currents. referring to major limitations of the proper operations of semiconductor devices (as CCDs and dynamic RAM memories), were examined by works [6]-[8].

\section{THEORETICAL MODEL}

The theoretical model of dark currents in CCDs was studied in detail by work [9]. Because for a CCD used in the multi-pinned phase (MPP) mode. the surface dark current generated at the $\mathrm{Si}-\mathrm{SiO}_{2}$ interface is almost completely suppressed. the main remaining sources of dark currents are the depletion (or bulk) current generated in the depletion region and the diffusion dark current from the field-free region.

In the region depleted of carriers (of size $x_{d e p}$. where the electric field sweeps the electrons and holes to the $n$ - and $p$-regions, resp.) of CCDs operating in the deep-depletion mode (when $n, p<<n_{i}$ ), the depletion dark current in $e^{-} / s$ (or counts/s) per pixel is: 


$$
D \bar{e}_{\text {dep }}=D e_{0, d e p}^{-} \cdot T^{3 / 2} \cdot \frac{\exp \left(-\frac{E_{g}}{2 k T}\right)}{\cosh \left[\frac{E_{t}-E_{i}}{k T}+a\right]},
$$

where:

$$
a=\arg \tanh \frac{\sigma_{p}-\sigma_{n}}{\sigma_{p}+\sigma_{n}},
$$

$D e_{0, d e p}=x_{d e p} A_{p i x} c_{n} v_{t h} N_{t} \frac{\sigma_{p} \sigma_{n}}{\sigma_{p}+\sigma_{n}} \exp \frac{\alpha}{k} \cosh a$

and: $c_{n}=\sqrt{N_{v} N_{c}} \cdot T^{-3 / 2}, \alpha=-\lim _{T \rightarrow 0 K} \frac{d E_{g}}{d T}$

$E_{g}$ being the energy gap, $E_{i}$ - the intrinsic Fermi level, $N_{t}$ - the concentration of bulk generationrecombination centers (traps) at the energy level $E_{t}$, $v_{t h}$ - the thermal velocity, $A_{p i x}$ - area of pixel, $\sigma_{p}$, $\sigma_{n}$ are the cross-sections for holes, and electrons, resp., while $N_{v}, N_{c}$ are the effective densities of states for the valence and conduction band, resp.

Similarly, the diffusion contribution to the dark currents is given by the expression [5], [9]:

$$
D \overline{d i f f}^{-D e_{0, d i f f}} \cdot T^{3} \cdot \exp \left(-\frac{E_{g}}{k T}\right),
$$

the pre-(exponential) factor of the diffusion dark

$$
\text { current being: } D e_{0, \text { diff }}=\frac{D_{n} A_{p i r} c_{n}^{2}}{x_{c} N_{a}},
$$

where $D_{n}$ is the diffusivity of electrons, from the

$$
\text { Fick's law: } \quad \bar{J}_{n}=-D_{n} \nabla n \text {, }
$$

$N_{a}$ is the concentration of acceptor impurities (in our case - boron), while the characteristic length $x_{c}=\inf \left(x_{f f}, L_{n}\right)$ is the least between the diffusion length of free electrons $L_{n}$ and the size $x_{f f}$ of the field-free region of CCD.

\section{CHARACTERISTIC ERRORS}

In order to evaluate (for the studied pixels) the material parameters (as $E_{g}, D e_{0, d e p}, D e_{0, \text { diff }}$. $\left|E_{t}-E_{i}\right|$, etc), we used the classical version [10]-[12] of the gradient method. Starting from the: a) most probable values $T_{i m p}, D_{i m p}$ of the temperature and of its associated dark current for each studied pixel, b) the square mean deviations $s\left(T_{i}\right), s\left(D_{i}\right)$ of the individual values $T_{i}, D_{i}$ corresponding to the state (temperature) $i$ and the correlation coefficient $r_{i}$ of these deviations, c) the values $T_{t i}, D_{t i}$ corresponding to the tangency point of the confidence ellipse $E_{i}$ to the plot $D e^{-}=f(T)$, there were calculated: (i) the reduced errors $z\left(T_{t i}\right), z\left(D_{t i}\right)$ for the tangency point:

$$
z\left(T_{t i}\right)=\frac{T_{t i}-T_{i m p}}{s\left(T_{i}\right)}, z\left(D_{t i}\right)=\frac{D_{t i}-D_{i m p}}{s\left(D_{i}\right)},
$$

(ii) the sum $S_{t i}$ of weighted squares of the reduced errors: $S_{t i}=z^{2}\left(T_{t i}\right)+z^{2}\left(D_{t i}\right)-2 r_{i} \cdot z\left(T_{t i}\right) \cdot z\left(D_{t i}\right)$, and: (iii) the error risk $q_{i}$ at the rejection of the compatibility of the theoretical relation $D e^{-}=f(T)$ relative to the state of individual values $\left(T_{i m p}, D_{i m p}\right)$ [13]:

$$
q_{i}=\exp \left(-\frac{S_{t i}}{2} \cdot\left(1-r_{i}^{2}\right)^{-1}\right)
$$

According to the usual procedures of mathematical statistics (see e.g. [1]-[3]), the compatibility of a theoretical relation relative to the state of individual values ( $T_{i m p}, D_{i m p}$ ) has to be rejected if the error risk $q_{i}$ is less than a certain limit (threshold) $L$, usually chosen between $0.001 \quad(=0.1 \%)$ and $0.02 \quad(=2 \%)$. Because even for the zero-order approximation of experimental errors (neglecting the rather important systematic errors), $56.25 \%$ of the theoretically predicted dark currents are compatible with the experimental results, we will assume a general compatibility, in the limits of the true total errors. It results that the real standard error [corresponding to the dark currents individual values with rejection error risks (calculated starting from the zero-order approximation of experimental errors) less than 0.001] has to be somewhat larger than that corresponding to the dispersion of the individual $D e^{-}$values. For $L=$ 0.001: $S_{t i}=\ln 10^{3} \cong 6.918$ (if $r_{i}=0$ ) and the first order approximation (lowest limit) of the average relative true errors (involving the systematic ones) will be the highest square mean deviation for all studied pixels and temperatures, ensuring (at limit) the compatibility of the theoretical model relative to the obtained experimental results:

$$
s(D e)=\sup \left\{\left|D_{t i}-D_{i m p}\right|\left[0.918-\left(\frac{T_{t i}-T_{i m p}}{s\left(T_{i}\right)}\right)\right]^{-1 / 2}\right\} .
$$

One obtains so that the minimal values of true (standard) experimental errors are: $1.12 \%$ (instead of $1.03 \%$ ) at $252 K, 0.71 \%$ (instead of $0.486 \%$ and $0.233 \%$, resp.) at $262 K$ and $271 K, 0.81 \%$ (instead of $0.233 \%$ ) at $281 K$, and $0.37 \%$ (instead of $0.297 \%$ ) at $291 K$. It results that the real average relative standard error corresponding to the 6 highest studied temperatures $(242 \ldots 291 K)$ has to be at least $1.608 \%$ (instead of the value $1.525 \%$ corresponding to the dispersion of individual values), value representing a first order approximation of the experimental errors. 


\section{PRE-EXPONENTIAL FACTORS AND MEYER-NELDEL RELATIONS}

Using the method of the regression line, we obtained the standard confidence intervals of Arrhenius' parameters: $\Delta E=0.92958 \pm 0.07597 \mathrm{eV}$ and:

$$
D \bar{e}_{0, A r r h}=(1.066 \ldots 465.4) \times 10^{17} \text { counts } / s \text {. }
$$

We obtained a very high value of the correlation coefficient between the natural logarithm of pre-factor $D e_{0, A r r h}$ and the activation energy $\Delta E: r_{A r r h .} \approx$ 0.9999177 , indicating so the validity of the MeyerNeldel rule: $\quad D e_{0}^{-}=D e_{00}^{-} \exp \left(\Delta E / E_{M N}\right)$,

with a value of the Meyer-Neldel energy:

$$
E_{M N} \cong 24.997 \mathrm{meV} \text {. }
$$

We studied also the compatibility of the theoretical model given by relations (2) [in the simplifying conditions of equal cross-sections: $\sigma_{p}=\sigma_{n} \rightarrow a=0$ and of deep-level traps located in proximity of the intrinsic Fermi level: $\left.E_{t} \cong E_{i}\right]$ and (5), obtaining the rather good (small) relative square mean error $2.722 \%$ corresponding to this theoretical description. After the elimination of $10 \%$ suspect (probably roughly erroneous) individual experimental data, the confidence intervals corresponding to the energy gap and to the pre-exponential factors of the diffusion and depletion terms resulted as: $E_{g}=1.0423 \pm 0.0256 \mathrm{eV}$,

$$
\begin{aligned}
D e_{0, d i f f}^{-} & =(3.77 \ldots 25.1) \times 10^{12} \text { counts } / s, \text { and: } \\
D e_{0, \text { depl }}^{-} & =(1.285 \ldots 2.948) \times 10^{6} \text { counts } / s, \text { resp. }
\end{aligned}
$$

Unfortunately, the existing theoretical methods for the evaluation of the sizes $x_{f f}, x_{d e p}$ of the free-field and of the depletion regions, resp. are too inaccurate to allow the comparison of the experimental values obtained for pre-factors $D e_{0, d i f f}^{-}, D e_{0, d e p}^{-}$with the corresponding theoretical predictions. That is why the experimental values of pre-factors are (conversely) used to evaluate the sizes of the field-free region $x_{f f}$ and of the depletion region $x_{d e p}$ [9].

While the correlation coefficients of the energy gap and pre-factors corresponding to the Arrhenius relation (1) and to the depletion term (2) of dark currents are mediocre: $r\left(E_{g}, \ln D e_{0, A r r h .}^{-}\right) \cong 0.81$ or even weak: $r\left(E_{g}, \ln D e_{0, d e p}^{-}\right) \cong 0.38 \ldots 0.72$, that of $E_{g}$ and of the diffusion term (5) of dark currents is very high: $r\left(E_{g}, \ln D e_{0, \text { diff }}^{-}\right) \approx 0.9998 \ldots 0.999992$ (depending on the studied pixels), indicating the validity of the Meyer-Neldel relation. In this last case, we obtained a value of the Meyer-Neldel energy of $25.3 \ldots 25.9 \mathrm{meV}$. The obtained results indicate a very weak correlation of $E_{g}$ with characteristic lengths $\left(x_{d e p}, x_{f f}, L_{n}\right)$, but (see also relation (6)) a probable very strong correlation of the electrons diffusivity $D_{n}$ with the energy gap, due to the migration entropy for the thermally activated jumping over potential barriers between different sites [4], [14]-[16].

\section{TEMPERATURE DEPENDENCE OF THE ENERGY GAP}

The specialty literature involves both linear and nonlinear semiempirical descriptions of the temperature dependence of the energy gap in Silicon. E.g., the graphical plot from Fig. 4.7 [17] is equivalent to the expression: $\quad E_{g}(\mathrm{eV})=1.20-3.4 \times 10^{-4} \mathrm{~T}$.

According to relations (4) from Section 3, this is equivalent to $\alpha / k \approx 3.947$ and the appearance of the factor $\exp \frac{\alpha}{k}(\cong 51.8)$ in expression (4) for $D e_{0, d e p}^{-}$.

As it concerns the nonlinear semiempirical relation of Sze [18]: $\quad E_{g}(\mathrm{eV})=1.17-\frac{4.73 \times 10^{-4} T^{2}}{T+636}$,

due to the presence of some very long "valleys" of the sum $S\left(D e_{0, \text { diff }}^{-}, D e_{0, d e p}^{-}, E_{g} ; T\right)$ of weighted squares of deviations of theoretical values of dark currents from their experimental values, the attempts to fit the parameters of nonlinear dependencies $E_{g}=f(T)$ starting from zero-order approximations (involving the assumptions: $\quad \sigma_{p}=\sigma_{n}$ and $E_{t} \cong E_{i}$ ) are not successful, because they lead to non-physical solutions [e.g. values up to $1.75 \mathrm{eV}$ for the energy gap in $\mathrm{Si}$ ].

Because the correction introduced by the factor $\cosh \left[\frac{E_{t}-E_{i}}{k T}\right]$ is more important for the depletion term (see relation (2)) of dark currents than the energy gap temperature dependence, the study of deep-level traps energies has to be accomplished previously.

\section{DEEP-LEVEL TRAPS}

We accomplished the fitting of the parameters of the theoretical model given by relations (2) [with the assumption $\sigma_{p}=\sigma_{n}$ ] and (5), obtaining the values of

parameters $D e_{0, d i f f}^{-}, D e_{(), d e p}^{-}, E_{g},\left|E_{t}-E_{i}\right|$. The average accuracy of this description $(2.36 \%)$ is better than that of the standard (simplified) description $(2.7225 \%)$ and rather near to the lowest limit of experimental errors $(1.608 \%)$. After the elimination of the roughly erroneous values, the remaining results grouped in the confidence intervals: 
$\left|E_{t}-E_{i}\right|=27.14 \pm 7.16 \mathrm{meV} \quad(11$ pixels $) \quad$ and $\left|E_{t}-E_{i}\right|=54.47 \pm 5.90 \mathrm{meV}$ (other 6 pixels), the results referring to the $18^{\text {th }}$ studied pixel converging towards $\left|E_{t}-E_{i}\right|=91.01 \mathrm{meV}$.

We compared these results with those from the specialty literature, synthesized by Table 1 . It results that the possible assignments of the obtained $\left|E_{t}-E_{i}\right|$ values are: $N i$. Co, $A u$ (or perhaps $P t$ ) for the interval $27.14 \pm 7.16 \mathrm{meV}, \mathrm{Mn}$ (or perhaps $P t$ ) for the interval $54.47 \pm 5.90 \mathrm{meV}, \mathrm{Fe}$, the unidentified trap 1 [25] (or perhaps $P t$ ) for $\left|E_{t}-E_{i}\right| \approx 91 \mathrm{meV}$. A more accurate evaluation of the efficiency (counts/ $\mathrm{e}$ ) of the studied CCDs will allow us to use some additional assignment criteria.

Table 1. Deep-level traps in Si and corresponding generation rates

\begin{tabular}{|c|c|c|c|}
\hline Trap & $\begin{array}{c}\left|E_{t}-E_{i}\right| \\
(\mathrm{meV})\end{array}$ & $\begin{array}{c}\text { Generation } \\
\text { rates }(\mathrm{e} / \mathrm{s} \text { at } \\
\left.55^{\circ} \mathrm{C}\right)\end{array}$ & $\begin{array}{c}\text { Refe- } \\
\text { rences }\end{array}$ \\
\hline$P t_{1}$ & 20 & & {$[23]$} \\
\hline$N i, \mathrm{Co}$ & $<30$ & 3700 & {$[25]$} \\
\hline$A u_{1}$ & $<30$ & 565 & {$[25]$} \\
\hline$M n$ & $\sim<50$ & 6400 & {$[25]$} \\
\hline$P t_{2}$ & 60 & 970 & {$[25]$} \\
\hline$P t_{3}$ & $90 \ldots 130$ & & {$[17]$,} \\
& & & {$[24]$} \\
\hline Trap 1 & 100 & 70 & {$[25]$} \\
\hline$F e$ & $120 \ldots 150$ & 195 & {$[25]$} \\
\hline
\end{tabular}

\section{CONCLUSIONS}

The good agreement of the obtained values of the deep-level traps energies with the experimental results obtained by means of different methods (as EPR [19], the deep-level transient spectroscopy [23], [24], the resistance $=\mathrm{f}(T)$ method [20], [21], etc), as well as the proximity of the average description error $(2.36 \%)$ of the theoretical model predictions relative to the lowest limit of experimental errors $(1.61 \%)$ indicate the high plausibility of the studied model. Some additional information concerning the $\sigma_{p}, \sigma_{n}$ cross-sections and the temperature dependence of the energy gap in Si are expected to be obtained in following, by means of the used dark current spectroscopy (DCS) method.

\section{References}

[1] W.T. Eadie, D. Drijard, F.E. James, M. Roos, B. Sadoulet, Statistical Methods in Experimental Physics, North-Holland Publishing Comp., Amsterdam - New York - Oxford, 1982.

[2] *** Handbook of Applicable Mathematics, chiefeditor W. Lederman, vol. 6 Statistics, John Wiley \& Sons, New York, 1984.
[3] P.W.M. John, Statistical Methods in Engineering and Quality Assurance, John Wiley \& Sons, 1990.

[4] R. Widenhorn, L. Münderman, A. Rest, E. Bodegom, J. Applied Physics, vol. 89, no.12, pp. 8179-8182. 2001.

[5] E. Bodegom, R. Widenhorn, D.A. Iordache, Proceedings of the $27^{\text {th }}$ International Semi-conductor Conference, October 4-6, 2004, Sinaia, vol. 2. pp. 363-366, 2004.

[6] J. van der Spiegcl, G.J. Declerck, Solid-State Electronics, vol. 27, no. 2, pp. 147-154, 1984.

[7] a) J.R. Janesick, T. Eliott, ASP Conf. Series, vol.23, p. 1, 1992; b) J.R. Janesick, Scientific ChargeCoupled Devices, SPIE, Bellingham, 2001.

[8] A. Rest, L. Münderman, R. Widenhorn, E. Bodegom, T.C. McGlinn, Rev. Sci. Instrum., vol. 73, no. 5, pp. 2028-2032, 20(02.

[9] E. Bodegom, R. Widenhorn "Interpreting Fitting Parameters", in Proc. 2" Colloquitum "Mathematics in Engineering and Numerical Physics" (MENP-2), April 2002, part 2, pp. 26-30, Credis Publishing House, Bucharest, 2004.

[10] K. Levenberg, Quart. Appl. Math., vol. 2, p. 164, 1944.

[11] D.W. Marquardt, Journal of Society Industr. Appl. Math., vol. 11, p. 431, 1963.

[12] Z. Mei, J.W. Morris jr., J. Nucl. Instr. Methods in Phys. Res., vol. 6, p. 371, 1990.

[13] D.A. Iordache "On the compatibility of some theoretical models relative to the experimental data", in Proc. 2"ul Colloquium "Mathematics in Engineering and Numerical Physics" (MENP-2), Bucharest, April 20()2, part 2, pp. 169-176, Credis Publishing House. Bucharest, 2004.

[14] E.R. Weber "Transition Metals in Silicon", Applied Physics A, vol. 30, pp. 1-22, 1983.

[15] M.S. Hu, in Atomic Diffusion in Semiconductors, D. Shaw edit., Plenum Press, New York, 1973.

[16] a) A. Yelon, B. Movaghar, Phys. Rev: Lett., vol. 65 , p. 618,1990 ; b) A. Yelon, B. Movaghar, H.M. Branz, Phis. Rer: B. vol. 46, p. 12244, 1992: c) G. Boisvert, L.J. Lewis, A. Yelon, Phys. Rev. Lett., vol. 75, p. 469,1995

[17] A.S. Grove, Phrsics and Technology of Semiconductor Devices, John Wilcy \& Sons, New York, 1967.

[18] S.M. S7e, Phisics of Semiconductor Devices, $2^{\text {nd }}$ edition. John Wiley \& Sons, New York, 1981.

[19] [19] H.H. Woodbury. G.W. Ludwig, P/lys. Rex., vol. 126, p. $466,1962$.

[20] H. Carchano, C. Jund, Solid-State Electron., vol. 13. p. 83,1970 .

[21] M. Conti, A. Panchicri, Alta Freq., vol. 40, p. 544. 1971.

[22] A.O. Evwaraye, E. Sun, J. Appl. Phys, vol. 47, p. 3172, 1976.

[23] Y.K. Kwon. T. Ishikawa, H. Kuwano. J. Appl. P/hx. vol. 61, no. 3, pp. 1055-1058, 1987.

[24] W.C. McColgin, J.P. Lavine, J. Kyan, D.N. Nichols, J. D. Russell, C. V. Stancampiano, in lefect Engineering in Semiconductor (ironth. Processing and Device Technology: S. Ashok, J. Chevalier, K. Sumino, E. Weber, eds. (Mater. Res. Soc. Proc., vol. 262, Pittsbugh, PA), pp. 769-774. 1992.

[25] W.C. McColgin, J.P. Lavine, C. V. Stancamplano, J.D. Russell, Mat. Res. Soc. Symp. Proc., vol. 510. pp. 475-480, 1998 . 